\title{
Simpaticectomía torácica bilateral por videocirugía. Diez años de experiencia
}

\author{
Leonardo Toscano*, Ulises Parada ${ }^{\dagger}$, Daniel Terra ${ }^{\ddagger}$, Siul Salisbury§, Juan Chifflet"
}

\section{Resumen}

Se define la hiperhidrosis como una excesiva sudoración en respuesta a estímulos térmicos o emocionales más allá de los requerimientos fisiológicos, con una incidencia de $2 \%$ a $4 \%$ en la población mundial. Si bien existen varios tipos de tratamientos, la opción quirúrgica brinda excelentes resultados con bajas tasas de recidiva.

Pacientes y método: estudio retrospectivo de 190 simpaticectomías torácicas videoasistidas realizadas desde 2003 hasta 2013, totalizando 95 pacientes. Se realizó una encuesta mediante un cuestionario sobre calidad de vida y sobre la severidad de los síntomas.

Resultados: se logró recabar el cuestionario en 58 pacientes (30\% hombres y $70 \%$ mujeres) con una media de edad de 25 años. El $90 \%$ se dio de alta entre las 24 y 48 horas del posoperatorio. Del total de pacientes, $72 \%$ presentaba hiperhidrosis severa. Del procedimiento podemos decir que dos pacientes presentaron recurrencia y un paciente refirió disconformidad por hipersequedad de la piel. El 80\% presentó algún tipo de hiperhidrosis compensadora, no siendo este un síntoma de preocupación mayor por parte de los pacientes. No hubo complicaciones de procedimiento. Respecto a la calidad de vida, era mala o regular en el $90 \%$ de los pacientes, mejorando en el posoperatorio, siendo buena o muy buena en el $95 \%$. El $94 \%$ de los pacientes están conformes o muy conformes con el procedimiento y sus resultados. Como conclusión, podemos establecer que la simpaticectomía torácica bilateral para el tratamiento de la hiperhidrosis es un método altamente seguro y efectivo.

Palabras clave: Hiperhidrosis

Simpatectomía

Cirugía torácica asistida por video

Key words: Hyperhidrosis

Sympathectomy

Video-assisted thoracic surgery

\footnotetext{
* Cirujano torácico, Hospital Central de las Fuerzas Armadas.

† Cirujano General. Asistente de la Clínica Quirúrgica "A".

¥ Cirujano torácico. Profesor Agregado del Depto. de Emergencia orientación Quirúrgica, Hospital de Clínicas.

$\S$ Cirujano torácico. Profesor Agregado de Cirugía de Tórax. Hospital de Clínicas.

T Cirujano torácico. Profesor Emérito de la Facultad de Medicina en Cirugía Torácica.

Correspondencia: Dr. Leonardo Toscano. Benito Blanco 626/405. Montevideo, Uruguay. Correo electrónico: Leonardo.toscano@vera.com.uy

Los autores declaran no tener conflictos de intereses.

Recibido: 13/9/18

Aprobado: 10/12/18
} 


\section{Introducción}

Se define la hiperhidrosis como una excesiva sudoración en respuesta a estímulos térmicos o emocionales más allá de los requerimientos fisiológicos. Mientras la sudoración normal brinda un mecanismo de homeostasis, enfriando el cuerpo por evaporación del sudor de la superficie de la piel. El exceso de sudoración lleva a alteraciones profundas en el plano social, emocional y laboral. La incidencia es muy variada de acuerdo a la zona geográfica, afectando entre $2 \%$ a $4 \%$ de la población mundial ${ }^{(1,2)}$, siendo más frecuente en países occidentales ${ }^{(1,3)}$. Existe una historia familiar en el $56,5 \%$ de los pacientes afectados ${ }^{(4)}$.

Si bien el tratamiento local, el sistémico y la psicoterapia han sido usados, la opción quirúrgica por videocirugía es de elección para los pacientes con hiperhidro$\operatorname{sis}^{(5-7)}$.

En el siguiente estudio se presenta la experiencia de un solo equipo quirúrgico en el tratamiento de la hiperhidrosis palmar y axilar por simpaticectomía realizada por videocirugía.

\section{Pacientes y método}

Se realizó un estudio retrospectivo de 190 simpaticectomías torácicas videoasistidas realizadas desde 2003 hasta 2013, totalizando 95 pacientes.

Se realizó un listado de los pacientes sometidos a cirugía. Se llamó a cada paciente, se les explicó el objetivo y metodología del estudio y se les solicitó completar el cuestionario de Milanez de Campos y colaboradores ${ }^{(8)}$, el cual es un interrogatorio de calidad de vida específico para hiperhidrosis validado en el $6^{\circ}$ Simposio Interna- cional de la Cirugía del Simpático en Viena en 2005 (tanto por vía telefónica como por correo electrónico), valorando la calidad de vida antes y después de la cirugía. Además, se evaluó la escala de severidad de hiperhidrosis (HDSS) ${ }^{(9)}$.

Las demás variables analizadas fueron la edad de inicio de los síntomas, edad al momento de la cirugía, grado de conformidad con el procedimiento quirúrgico, aparición de hiperhidrosis compensadora en relación con la severidad y la localización. A todos los pacientes se les realizó el mismo procedimiento (simpaticectomía torácica bilateral por videocirugía) por el mismo equipo de cirujanos en diferentes centros de atención, tanto públicos como privados. Fue importante determinar el grado de severidad de la hiperhidrosis en cuanto a la afectación de la calidad de vida del paciente.

El procedimiento consistió, en todos los casos, en anestesia general e intubación selectiva izquierda. El paciente fue colocado en decúbito dorsal, semisentado a $60^{\circ}$, con los brazos extendidos en abducción a $90^{\circ}$, cuidando de no hiperextender el plexo braquial y fijándolo con un sistema acolchonado. Se realizaron tres abordajes torácicos: uno de $10 \mathrm{~mm}$ en el quinto espacio intercostal en la línea axilar anterior y dos de $5 \mathrm{~mm}$ en el tercer espacio intercostal en la línea axilar media y posterior (figura 1).

Se utilizó una cámara de $0^{\circ}$ en todos los casos. Se identificó la cadena simpática entre el borde inferior de la segunda costilla y el borde superior de la quinta costilla (que incluye los ganglios T2-T4) (figuras 2 y 3 ), apertura de la pleura parietal (figura 4) y simpaticectomía cuidando de no lesionar las estructuras subyacentes e identificar posibles ramos de Kuntz.

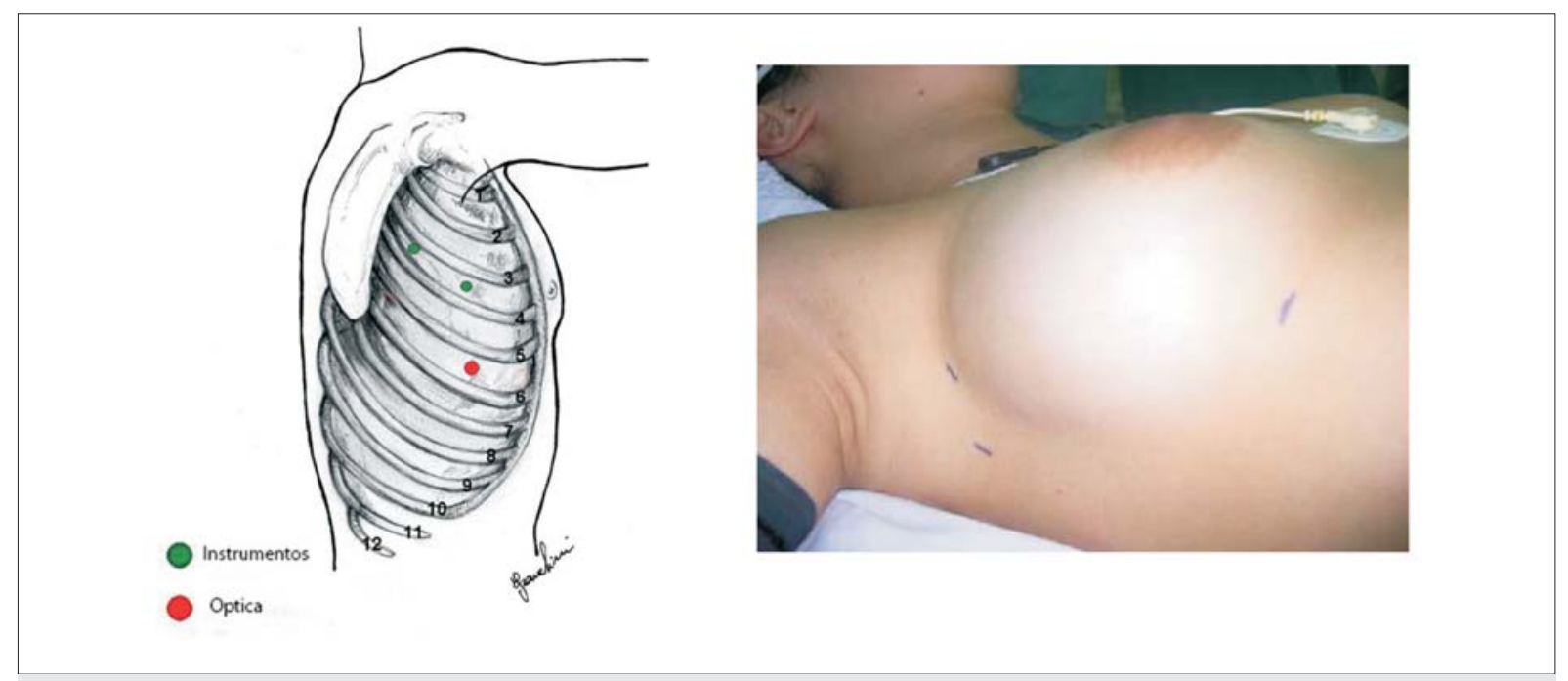

Figura 1. Sitio de inserción de los trócares. En rojo: $10 \mathrm{~mm}$ para endocámara quinto espacio intercostal LAA. En verde: dos trócares de $5 \mathrm{~mm}$ en tercer espacio intercostal. 


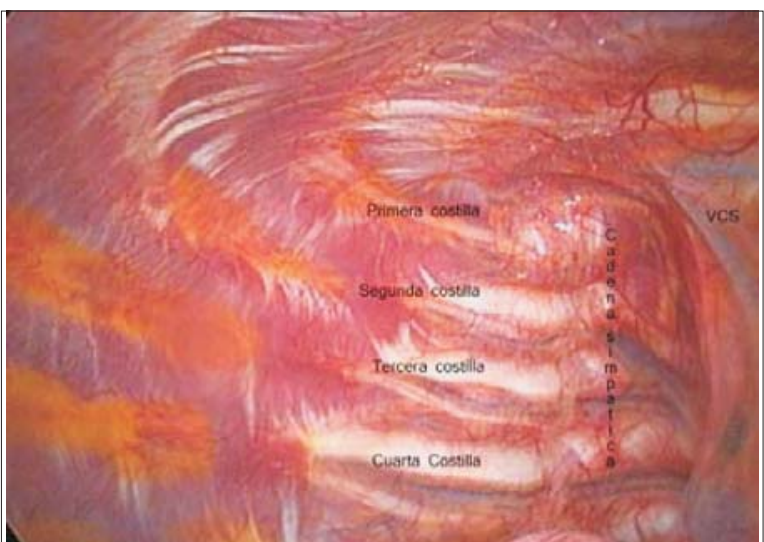

Figura 2. Visión endoscópica del sector superior del hemitórax derecho. Se identifica: vena cava superior (VCS), primera, segunda, tercera y cuarta costilla, cadena simpática

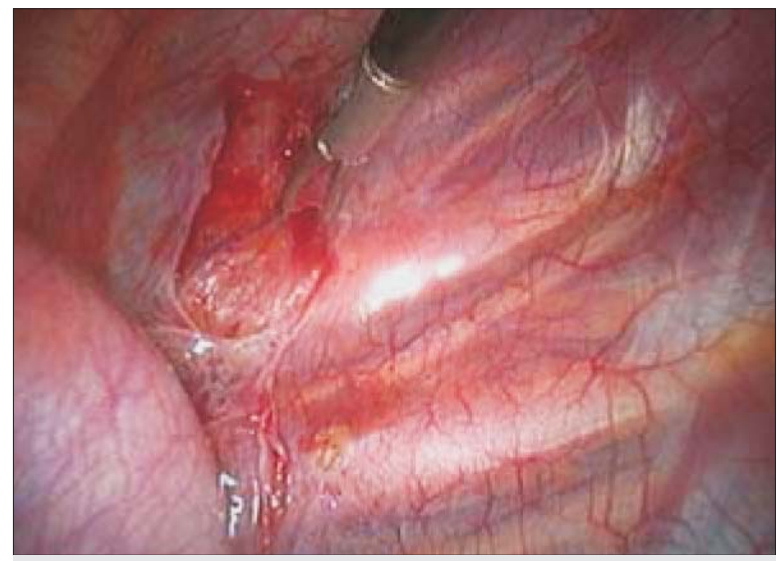

Figura 4. Apertura de la pleura parietal sobre la cadena simpática desde el borde inferior de la segunda costilla hasta el borde superior de la quinta costilla

Para la resección del simpático en un inicio se utilizó electrobisturí, pero en los últimos cuatro años el procedimiento se viene realizando con bisturí armónico o con Ligasure ${ }^{\circledR}$ (figura 5). Se comprobó la reexpansión pulmonar bajo visión directa. Vale la pena recalcar que ningún caso requirió toracotomía.

Se infiltraron las incisiones con bupivacaína a $0,25 \%$. Se dejaron en todos los casos drenajes pleurales finos conectados a sistema con sello de agua, siendo inicialmente de 14 o 16 Fr y actualmente de 10 Fr. Estos se retiraron a las 24 horas con control radiológico previo y los pacientes fueron dados de alta entre las 24 y 48 horas del posoperatorio.

Se usaron analgésicos mayores, como tramadol, a dosis de $100 \mathrm{mg}$ cada ocho horas en el posoperatorio in-

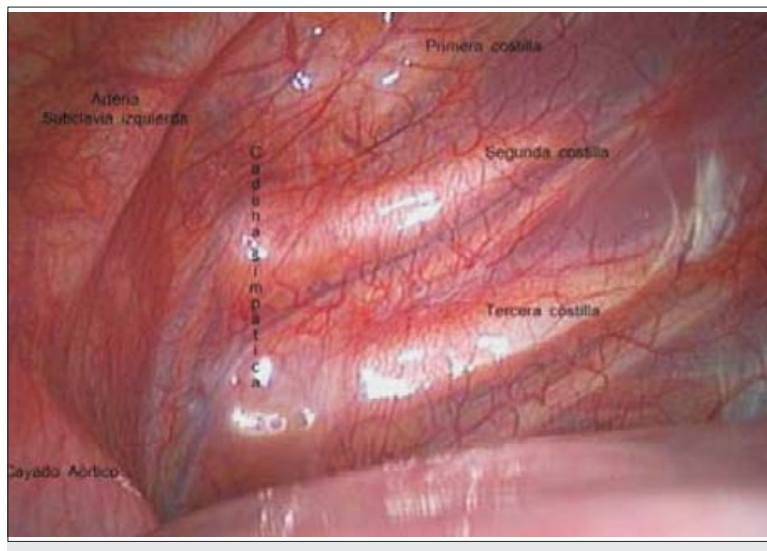

Figura 3. Visión endoscópica del sector superior del hemitórax izquierdo.Se identifica: arteria subclavia izquierda, primera, segunda y tercera costilla, cayado aórtico y cadena simpática. Desde este hemitórax el trocar para endocámara debe ser posicionado más lateral para mejorar la visión por la presencia de la aorta.

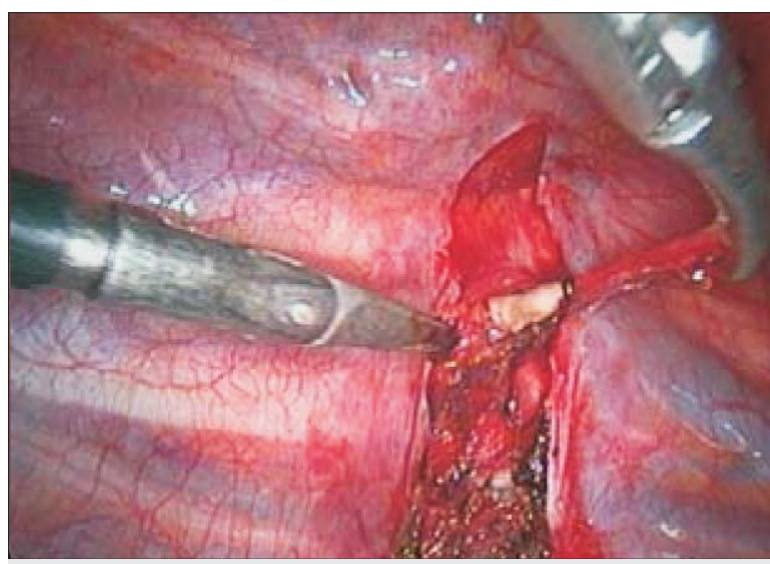

Figura 5. Resección del simpático con bisturi armónico o Ligasure y envío a anatomía patológica.

mediato por vía intravenosa y a las 48 horas, al momento del alta, se utilizó el mismo analgésico por vía oral por una semana aproximadamente. Los pacientes volvieron a sus tareas habituales entre el cuarto y séptimo día del posoperatorio.

\section{Resultados}

De los 95 pacientes operados se logró recabar el cuestionario en 58, por lo que se excluyen del análisis estadístico 37 pacientes, una paciente planteó su disconformidad y se negó a completar el cuestionario y el resto no fue posible localizarlo.

El 30\% fueron hombres y el $70 \%$ restante correspondió a pacientes del sexo femenino. La edad al momento de la cirugía fue de 25 años (13-47), con una media de edad de inicio de los síntomas a los 10 años (4-29). En 


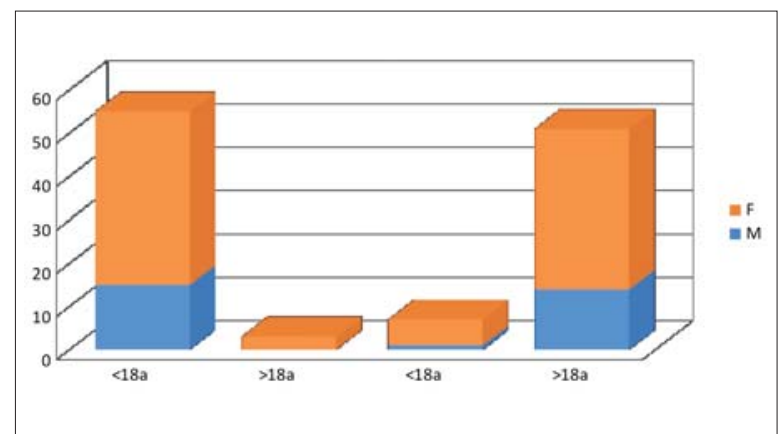

Figura 6. Edad de inicio de los síntomas por sexo.

todos los casos correspondió a pacientes con hiperhidrosis palmar, axilar o combinación de ambas (figura 6).

No hubo ninguna complicación intra ni posoperatoria en toda la serie. En todos los casos se comprobó que las manos de los pacientes estaban secas y calientes en el intraoperatorio una vez culminado el procedimiento. El $90 \%$ de los pacientes fueron dados de alta antes de las 48 horas del posoperatorio con analgésicos vía oral. El seguimiento fue por un mínimo de dos años en consulta cada seis meses en la mayoría de los casos.

El 72\% (42) de los pacientes operados refirieron tener una hiperhidrosis severa según la escala de hiperhidrosis de 4 puntos. El 20,7\% tenía un puntaje de 3 (12 pacientes), dos pacientes tenían un puntaje de $2(3,4 \%)$ y dos pacientes un puntaje de $1(3,4 \%)$ (tabla 1$)$.

Se presentó recurrencia de los síntomas en dos pacientes $(3,4 \%)$. Ambos casos requirieron una reintervención, ascendiendo en la resección sobre la cadena simpática. Uno de los pacientes quedó conforme luego del procedimiento y el otro refirió disconformidad por hipersequedad de la piel $(1,7 \%)$.

En cuanto a la hiperhidrosis compensadora se presentó en el $80 \%$ de los pacientes, siendo en una sola topografía en $38 \%$ de los casos y en múltiples sitios en el $62 \%$. De los sitios donde se presentó la hiperhidrosis compensadora, el dorso fue el más frecuente con $75 \%$, el abdomen $50 \%$, en los muslos $30 \%$ y en los pies $16 \%$ (tabla 2). Solo dos pacientes hicieron referencia a hiperhidrosis compensadora relacionada con el clima (con los aumentos de temperatura ambiental). En ningún caso la hiperhidrosis compensadora resultó un problema mayor que la hiperhidrosis axilar o palmar. No hubo casos de síndrome de Horner en nuestra serie.

Con respecto a la calidad de vida antes y después del procedimiento, previo a la cirugía era mala o muy mala en $50 \%$ de los pacientes, regular en $39,7 \%$ y buena o muy buena en $10,3 \%$. En el posoperatorio la calidad de vida mejoró, siendo buena o muy buena en $94,8 \%$ de los pacientes y permaneciendo mala o muy mala en 3,4\% (figura 7 ).
Tabla 1. Escala de severidad de la hiperhidrosis (HDSS).

\begin{tabular}{ll}
\hline 1 & 42 pacientes $(72,4 \%)$ \\
2 & 12 pacientes $(20,7 \%)$ \\
3 & 2 pacientes $(3,4 \%)$ \\
4 & 2 pacientes $(3,4 \%)$ \\
\hline
\end{tabular}

Tabla 2. Evaluación de la calidad de vida antes y después de la cirugía.

\begin{tabular}{|ccc|}
\hline $\begin{array}{c}\text { Puntaje de calidad } \\
\text { de vida }\end{array}$ & $\begin{array}{c}N^{0} \text { de pacientes antes } \\
\text { de la cirugía }\end{array}$ & $\begin{array}{c}N^{0} \text { de pacientes } \\
\text { después de la cirugía }\end{array}$ \\
\hline 1 (muy mala) & 8 & 2 \\
2 (mala) & 21 & 0 \\
3 (regular) & 23 & 1 \\
4 (buena) & 5 & 10 \\
5 (muy buena) & 1 & 45 \\
\hline
\end{tabular}

Con respecto a la interferencia de la hiperhidrosis sobre las cuestiones de la vida diaria, se evaluó sobre un total de 100 puntos: el $46,5 \%$ de los pacientes tuvo un cambio en más de 50 puntos; $46,5 \%$ de 20 a 49 puntos, y $6,8 \%$ menos de 20 puntos.

En cuanto a la conformidad global con el procedimiento, el $94 \%$ de los pacientes está conforme o muy conforme con los resultados y solo $6 \%$ está disconforme (tabla 3).

\section{Discusión}

La hiperhidrosis palmar y axilar es un problema que, según las estadísticas, afecta a unos 115.000 uruguayos, que lleva a problemas sociales, laborales y a interrumpir las actividades diarias ${ }^{(4,10)}$. Si bien existen tratamientos no quirúrgicos, estos tienen corta acción y efectos secundarios.

La simpaticectomía torácica bilateral por videocirugía es efectiva y segura si es realizada por cirujanos entrenados tanto para la hiperhidrosis palmar como la axilar, con muy baja morbimortalidad, resultados cosméticos satisfactorios, escaso dolor en el posoperatorio, corta hospitalización y rápido reintegro a las actividades diarias ${ }^{(4)}$.

El éxito de la videocirugía varía entre $88 \%$ y $100 \%$ según las series ${ }^{(4,7,10)}$. En nuestro estudio se observó una 


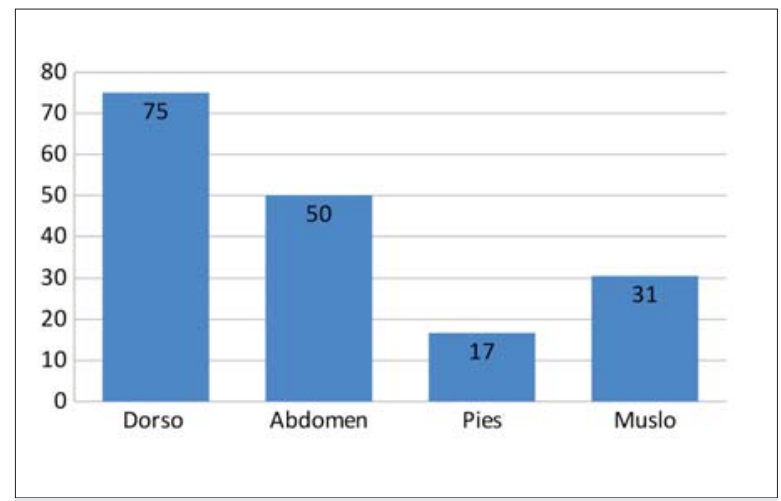

Figura 7. Distribución de hiperhidrosis compensadora

mejoría de los síntomas en el 95\% de los pacientes luego del procedimiento, con un grado de satisfacción también de $95 \%$. El 5\% de los pacientes disconformes está de acuerdo con el porcentaje de pacientes con hiperhidrosis leve que decidieron operarse, lo que es acorde con lo que muestran los datos internacionales respecto a que los pacientes con menos síntomas son los menos beneficiados de la cirugía ${ }^{(11,12)}$.

Es importante destacar que la mayoría de nuestros pacientes se operaron a los 25 años, pero que venían padeciendo esta enfermedad por diez años en promedio. Este dato es de gran importancia, ya que la mayoría de los pacientes había tenido consultas médicas en ese lapso, no teniendo respuesta por parte de los profesionales tratantes en cuanto a las opciones de tratamiento o los tratamientos previos no fueron efectivos.

Desde el punto de vista del procedimiento, si bien hay autores que realizan la cirugía con tubo orotraqueal simple, nosotros pensamos que la utilización de tubos de doble luz mejoran la exposición del campo operatorio y disminuyen el riesgo de lesión parenquimatosa durante la introducción de los trócares. El cambio del uso de electrobisturí a bisturí armónico o Ligasure ${ }^{\circledR}$ disminuyó la posibilidad de lesión de estructuras cercanas a la cadena simpática durante el procedimiento y además significó una mejoría en los niveles de dolor de los pacientes, disminuyendo los requerimientos de analgesia posoperatoria.

No hay diferencias significativas en cuanto a la mejoría de la hiperhidrosis comparando el clipado y la sección de este. El beneficio aparente de reversibilidad del clipado ante resultados no satisfactorios no está del todo demostrado, existiendo pocos casos en la literatura y siendo necesario retirar los clips en un período de dos semanas luego de la cirugía, no siendo suficiente para que el paciente evalúe la mejoría de los síntomas ${ }^{(3)}$. Las críticas que tenemos es la posibilidad de falla del procedimiento por falta de prensión del clip o caída del mismo,
Tabla 3. Grado de satisfacción con la cirugía

\begin{tabular}{ll}
\hline Mucho mejor (5) & 44 pacientes $(75 \%)$ \\
Mejor (4) & 11 pacientes $(19 \%)$ \\
Igual (3) & 0 \\
Peor (2) & 2 pacientes $(3,5 \%)$ \\
Mucho peor (1) & 1 paciente $(2,5 \%)$ \\
\hline
\end{tabular}

por lo que preferimos la resección de la cadena simpática.

En cuanto a la extensión de la resección, optamos por resecar T2-T4, ya que ha demostrado los mayores beneficios $^{(13,14)}$ y un porcentaje aceptable de hiperhidrosis compensadora ${ }^{(14-17)}$.

En nuestra serie se presentó $80 \%$ de hiperhidrosis compensadora, lo cual es acorde a la literatura internacional $^{(4,18)}$, pero en ningún caso este fenómeno indeseado alteró la satisfacción del paciente con el procedimiento realizado, pues en el 100\% de los casos es leve.

La falla de procedimiento se evidencia tanto por la reaparición de los síntomas como por la disconformidad del paciente con el procedimiento. En la primera, la falta de sección de los nervios de Kuntz es la principal causa de recidiva. La disconformidad con el procedimiento se observa en pacientes que no tenían una gran repercusión en su vida diaria causada por la hiperhidrosis ${ }^{(18,19)}$. En nuestra serie, dos pacientes (5\%) tuvieron una falla de procedimiento.

\section{Conclusión}

La simpaticectomía torácica por videocirugía es un procedimiento altamente efectivo y seguro (con equipos quirúrgicos entrenados) para pacientes con hiperhidrosis palmar y axilar severa, con altas tasas de satisfacción por parte de los pacientes, mejorando significativamente la calidad de vida de estos.

\section{Abstract}

Hyperhidrosis is defined as excessive sweating as a response of thermal or emotional stimuli beyond physiological requirements, and it incidence is 2 to $4 \%$ of the global population. In spite of there being several kinds of treatment, surgery provides excellent results with low relapse rates.

Method: retrospective study of 190 video-assisted thoracic sympathectomies performed from 2003 until 2013, accounting for 95 patients. A survey was conduc- 
ted through a quality-of-life questionnaire and questions on the severity of symptoms.

Results: 58 patients completed the survey $(30 \%$ men and $70 \%$ women) with an average age of 25 years old. $90 \%$ were discharged from hospital between 24 and 48 hours after surgery. $72 \%$ of patients presented severe hyperhidrosis. Based on the procedure, we may state 2 patients presented recurrence and only one patient declared to be uncomfortable with the hyper-dryness of skin. $80 \%$ of patients presented some kind of compensating hyperdidrosis, being the no reason for concern among patients. There were no complications arising from the procedure. As to the quality of life it used to be bad or fair in $90 \%$ of patients, and improved in the postoperative period, being it good or very good in $95 \%$ of them. $94 \%$ of patients are satisfied or very satisfied with the procedure and its results. As a conclusion, we may sat that bilateral thoracic sympathectomy is a highly safe and effective method to treat hyperhidrosis.

\section{Resumo}

A hiperidrose está definida como uma sudoração excessiva como resposta a estímulos térmicos ou emocionais superior aos requerimentos fisiológicos; tem uma incidência de 2 a 4\% na população mundial. Embora vários tipos de tratamentos estejam disponíveis, a cirurgia tem resultados excelentes com baixas taxas de recidiva.

Pacientes e métodos: estudo retrospectivo de 190 simpatectomias torácicas videoassistidas realizadas no período 2003-2013 em um total de 95 pacientes. Realizou-se um questionário sobre qualidade de vida e gravidade dos sintomas.

Resultados: 58 pacientes com idade média de 25 anos responderam o questionário (30\% homens e 70\% mulheres). $90 \%$ teve alta entre 24 e 48 hs pós cirurgia. $72 \%$ dos pacientes apresentava hiperidrose severa. Com relação ao procedimento 2 pacientes apresentaram recorrência e 1 paciente declarou não estar satisfeita devido ao ressecamento excessivo da pele. $80 \%$ apresentou algum tipo de hiperidrose compensadora, não sendo este um sintoma de maior preocupação dos pacientes. Não foram registradas complicações relacionadas ao procedimento. Em 90\% dos pacientes a qualidade de vida era ruim ou regular melhorando no pós-operatório, a boa ou muito boa em $95 \%$ dos casos. $94 \%$ dos pacientes relatou estar satisfeito ou muito satisfeito com o procedimento e seus resultados. Como conclusão podemos dizer que a simpatectomia torácica bilateral para o tratamento da hiperidrose é um método muito seguro e efetivo.

\section{Bibliografía}

1. Strutton DR, Kowalski JW, Glaser DA, Stang PE. US prevalence of hyperhidrosis and impact on individuals with axi- llary hyperhidrosis: results from a national survey. J Am Acad Dermatol 2004; 51(2):241-8.

2. Shargall Y, Spratt E, Zeldin RA. Hyperhidrosis: what is it and why does it occur? Thorac Surg Clin 2008; 18(2):125-32.

3. Lin CC, Mo LR, Lee LS, Ng SM, Hwang MH. Thoracoscopic T2-sympathetic block by clipping - a better and reversible operation for treatment of hyperhidrosis palmaris: experience with 326 cases. Eur J Surg Suppl 1998; 580:13-6.

4. de Campos JR, Kauffman P, Werebe Ede C, Andrade Filho LO, Kusniek S, Wolosker N, et al. Quality of life, before and after thoracic sympathectomy: report on 378 operated patients. Ann Thorac Surg 2003; 76(3):886-91.

5. Baumgartner FJ, Bertin S, Konecny J. Superiority of thoracoscopic sympathectomy over medical management for the palmoplantar subset of severe hyperhidrosis. Ann Vasc Surg 2009; 23(1):1-7.

6. Ishy A, de Campos JR, Wolosker N, Kauffman P, Tedde ML, Chiavoni CR, et al. Objective evaluation of patients with palmar hyperhidrosis submitted to two levels of sympathectomy: T3 and T4. Interact Cardiovasc Thorac Surg 2011; 12(4):545-8.

7. Rodríguez PM, Freixinet JL, Hussein M, Valencia JM, Gil RM, Herrero J, et al. Side effects, complications and outcome of thoracoscopic sympathectomy for palmar and axillary hyperhidrosis in 406 patients. Eur J Cardiothorac Surg 2008; 34(3):514-9.

8. Panhofer P, Naumayer C, Zacherl J, JAkesz R, Bischof G. A survey and validation guide for health-related quality-of-life status in surgical treatment of hyperhidrosis. Eur Surg 2005; 37(3):143-52.

9. Solish N, Bertucci V, Dansereau A, Hong HC, Lynde C, Lupin M, et al. A comprehensive approach to the recognition, diagnosis, and severity-based treatment of focal hyperhidrosis: recommendations of the Canadian Hyperhidrosis Advisory Committee. Dermatol Surg 2007; 33(8):908-23.

10. Munia MA, Wolosker N, Kauffman P, de Campos JR, Puech-Leão P. A randomized trial of T3-T4 versus T4 sympathectomy for isolated axillary hyperhidrosis. J Vasc Surg 2007; 45(1):130-3.

11. Kumagai K, Kawase H, Kawanishi M. Health-related quality of life after thoracoscopic sympathectomy for palmar hyperhidrosis. Ann Thorac Surg 2005; 80(2):461-6.

12. Baumgartner FJ, Toh Y. Severe hyperhidrosis: clinical features and current thoracoscopic surgical management. Ann Thorac Surg 2003; 76(6):1878-83.

13. Schmidt J, Bechara FG, Altmeyer P, Zirngibl H. Endoscopic thoracic sympathectomy for severe hyperhidrosis: impact of restrictive denervation on compensatory sweating. Ann Thorac Surg 2006; 81(3):1048-55.

14. Dewey TM, Herbert MA, Hill SL, Prince SL, Mack MJ. One-year follow-up after thoracoscopic sympathectomy for hyperhidrosis: outcomes and consequences. Ann Thorac Surg 2006; 81(4):1227-32.

15. Yazbek G, Wolosker N, de Campos JR, Kauffman P, Ishy A, Puech-Leão P. Palmar hyperhidrosis - which is the best 
level of denervation using video-assisted thoracoscopic sympathectomy: T2 or T3 ganglion? J Vasc Surg 2005; 42(2):281-5.

16. Jaffer U, Weedon K, Cameron AE. Factors affecting outcome following endoscopic thoracic sympathectomy. Br J Surg 2007; 94(9):1108-12.

17. Reisfeld R, Nguyen R, Pnini A. Endoscopic thoracic sympathectomy for hyperhidrosis: experience with both cauterization and clamping methods. Surg Laparosc Endosc Percutan Tech 2002; 12(4):255-67.
18. Campanati A, Penna L, Guzzo T, Menotta L, Silvestri B, Lagalla G, et al. Quality-of-life assessment in patients with hyperhidrosis before and after treatment with botulinum toxin: results of an open-label study. Clin Ther 2003; 25(1):298-308.

19. Amir M, Arish A, Weinstein Y, Pfeffer M, Levy Y. Impairment in quality of life among patients seeking surgery for hyperhidrosis (excessive sweating): preliminary results. Isr J Psychiatry Relat Sci 2000; 37(1):25-31. 